\title{
Effect of uric acid-lowering therapy on renal function in patients with chronic kidney disease: a systematic review and meta-analysis
}

Yukio Maruyama ${ }^{1 *}(0)$, Takanori Kumagai ${ }^{2}$, Naoki Sugano ${ }^{1}$, Shigetaka Yoshida ${ }^{3}$, Kimiyoshi Ichida ${ }^{4}$ and Shunya Uchida ${ }^{5}$

\begin{abstract}
Background: Whether uric acid (UA)-lowering therapy (ULT) is effective in reducing the progression of renal dysfunction in patients with chronic kidney disease (CKD) remains controversial. Since several advances have been made in therapies for hyperuricemia, including novel xanthine oxidoreductase (XOR) inhibitors, we conducted a systematic review to clarify the effectiveness of ULT in preserving renal function among CKD patients.

Methods: In this systematic review, the MEDLINE database was searched up to June 2019. We included complete randomized controlled trials comparing renal events between adult non-dialyzed CKD patients, defined as estimated glomerular filtration rate (eGFR) $<60 \mathrm{~mL} / \mathrm{min} / 1.73 \mathrm{~m}^{2}$, with and without ULTs. Changes in eGFR were expressed as the mean difference (MD). The incidence of dichotomous outcomes was expressed as a risk ratio. This review was performed using the predefined protocol published in PROSPERO (CRD 42019140346).

Results: Eleven studies with 4277 CKD patients were included. Drugs used in the intervention groups of all studies were XOR inhibitors (allopurinol, febuxostat or topiroxostat). Although patients with ULT tended to show superior preservation of eGFR as compared to those without ULT, no significant differences were identified (MD, 2.52; 95\% confidence interval, -0.15 to 5.18 ). In subgroup analysis, the use of allopurinol was associated with superior preservation of eGFR, whereas the newer XOR inhibitors, febuxostat and topiroxostat, showed no significant effects on eGFR changes. Neither incidence of end-stage kidney disease nor treatment-emergent adverse events differed significantly between groups.
\end{abstract}

Conclusions: The present systematic review and meta-analysis suggested that CKD patients with ULT tend to show superior eGFR preservation as compared to patients without ULT, but further studies are needed to verify the renoprotective effects of ULT.

Keywords: Allopurinol, Febuxostat, Renoprotective effect, Topiroxostat, Xanthine oxidoreductase inhibitor

\footnotetext{
*Correspondence: maruyama@td5.so-net.ne.jp

${ }^{1}$ Division of Nephrology and Hypertension, Department of Internal

Medicine, The Jikei University School of Medicine, 3-19-18 Nishi-shinbashi,

Minato-ku, Tokyo 105-8471, Japan

Full list of author information is available at the end of the article
}

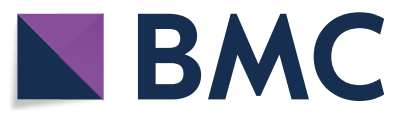

(c) The Author(s) 2021. Open Access This article is licensed under a Creative Commons Attribution 4.0 International License, which permits use, sharing, adaptation, distribution and reproduction in any medium or format, as long as you give appropriate credit to the original author(s) and the source, provide a link to the Creative Commons licence, and indicate if changes were made. The images or other third party material in this article are included in the article's Creative Commons licence, unless indicated otherwise in a credit line to the material. If material is not included in the article's Creative Commons licence and your intended use is not permitted by statutory regulation or exceeds the permitted use, you will need to obtain permission directly from the copyright holder. To view a copy of this licence, visit http://creativecommons.org/licenses/by/4.0/. The Creative Commons Public Domain Dedication waiver (http://creativecommons.org/publicdomain/zero/1.0/) applies to the data made available in this article, unless otherwise stated in a credit line to the data. 


\section{Introduction}

Asymptomatic hyperuricemia is frequently associated with a higher mortality rate and a wide variety of risk factors for hypertension, metabolic syndrome, coronary artery disease, cardiovascular disease (CVD), vascular dementia, preeclampsia and kidney disease [1]. In particular, serum concentrations of uric acid (UA) are commonly elevated in patients with chronic kidney disease (CKD), as a result of decreased renal function. Feig et al. [2] summarized the results of 12 observational studies and found that 7 studies suggested an association between higher serum UA and the prevalence and progression of CKD, whereas four did not. Among these, Hsu et al. [3] reported that patients in the highest quartile of serum UA had a hazard ratio of 2.14 for the development of end-stage kidney disease (ESKD), after adjusting for other risk factors. They used the US Renal Data System database of 177,570 patients, followed over 25 years. Because of the number of confounders in assessments of the effects of serum UA on patient outcome, whether hyperuricemia is a cause or a result of CKD (in other words, whether serum UA independently increases the risk of CKD) remains unclear.

Several randomized controlled trials (RCTs) and systematic (SRs) reviews have evaluated the efficacy of UA reductions on progression of CKD. Sampson et al. [4] conducted a SR of 12 RCTs and 1,187 participants, and found that estimated glomerular filtration rate (eGFR) was probably increased with UA-lowering therapy (ULT) at six months and one year, but probably makes little or no difference at two or five years. On the other hand, Bose et al. [5] failed to identify any renoprotective effects of allopurinol in a SR of eight RCTs including 476 participants. Several SRs have targeted CKD patients, showing improvements in eGFR among ULT users [6, 7]. It is noteworthy that CKD was defined according to the guidelines or by study authors in these SRs, and the presence of diabetes, proteinuria and serum creatinine in addition to eGFR were taken into account for diagnosis. Therefore, they included patients with eGFR both higher and lower than $60 \mathrm{~mL} / \mathrm{min} / 1.73 \mathrm{~m}^{2}$. However, eGFR is thought to be most important factors in the management of CKD because it is well known that both strongly influence on patient outcome. Additionally, a dose adjustment for allopurinol, most commonly used ULT, is needed based on eGFR. In addition to allopurinol, febuxostat and topiroxostat became available in Japan in 2011 and 2013, respectively. These newer xanthine oxidoreductase (XOR) inhibitors have a non-purine structure and are mainly metabolized in the liver, then excreted in both the urine and feces. Accordingly, their impacts on the kidney are minimal, and dose adjustment depending on renal function is not necessarily required, unlike allopurinol.
These drugs are thus expected to be fully administrable to compromised CKD patients and thus to be more effective than allopurinol in this population. Lin et al. [8] conducted a SR revealing a rise in eGFR in febuxostat users as compared to controls among CKD stage 3 and 4 patients. However, this SR also included RCT of dialyzed patients or patients with eGFR higher than $60 \mathrm{~mL} /$ $\mathrm{min} / 1.73 \mathrm{~m}^{2}$.

Since more data have been accumulated regarding the clinical utility of newer XOR inhibitors, including febuxostat and topiroxostat, we conducted a SR of studies published up to 2019, to clarify the effectiveness of ULT among patients with CKD, defined as patients with eGFR $<60 \mathrm{~mL} / \mathrm{min} / 1.73 \mathrm{~m}^{2}$.

\section{Materials and methods}

SRs and meta-analyses were undertaken according to the Preferred Reporting Items for Systematic Reviews and Meta-Analyses (PRISMA) guidelines [9] (Additional file 1). This review was performed using the predefined protocol published in PROSPERO (CRD42019140346). No ethics approval was required, as this study did not include confidential personal data and did not involve patient interventions.

The MEDLINE database was searched for relevant articles up to June 2019, with no restrictions on language, time or methodology, through focused and highly sensitive search strategies (Additional file 2). After excluding duplicates, we imported citations, then title and abstract were screened independently by four researchers (Y.M., T.K., N.S. and S.Y.). After the first screening, relevant studies were retrieved independently by the same researchers in full text to check for eligibility and whether specific inclusion/exclusion criteria were met. All disagreements were resolved as consensus decisions of all four researchers.

We included complete RCTs comparing renal events between adult non-dialyzed CKD patients on ULT and those without ULT. CKD was defined as an eGFR $<60 \mathrm{~mL} / \mathrm{min} / 1.73 \mathrm{~m}^{2}$. In studies including patients with eGFR both higher and lower than $60 \mathrm{~mL} / \mathrm{min} / 1.73 \mathrm{~m}^{2}$, we extracted the data of only patients with lower eGFR. In studies where required data were not available, the corresponding authors were contacted by email. Exclusion criteria were studies including patients with normal renal function, patients $<18$ years old, different outcomes, comparisons between different ULTs, reviews and study protocols. Outcomes of interest were the changes in renal function as assessed by eGFR, incidence of ESKD and adverse events. ESKD was defined as the initiation of renal replacement therapy. Adverse events were assessed as treatment-emergent adverse events (TEAEs) leading to study drug discontinuation. If the necessary data were 


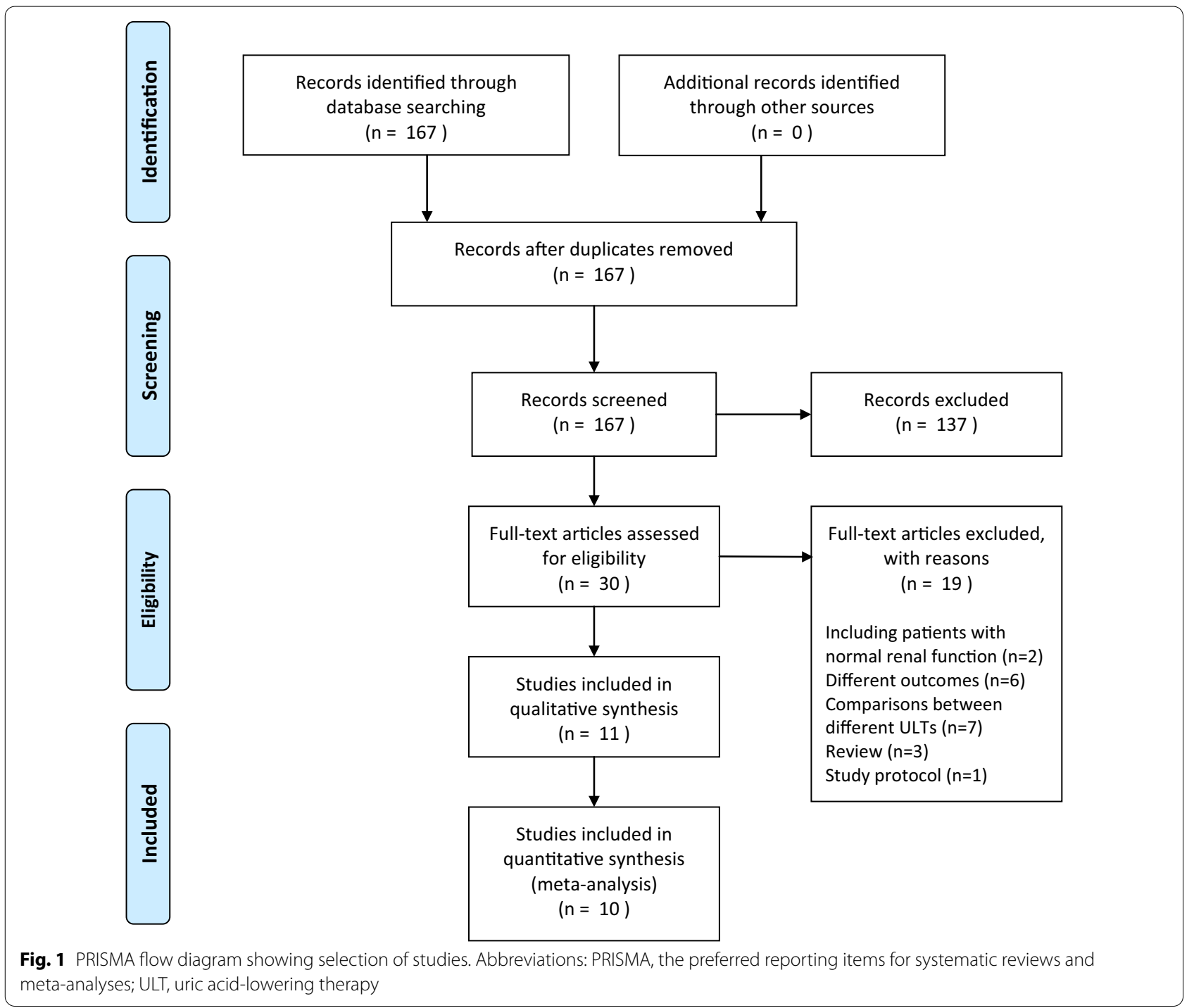

not reported, we contacted the corresponding author to obtain this information.

\section{Quality assessment}

The same four researchers (Y.M., T.K., N.S. and S.Y.) independently assessed the risk of bias for each study using the risk of bias assessment tool from the Cochrane Handbook for Systematic Reviews of Intervention. We assessed random sequence generation, allocation concealment, blinding, incomplete outcome data, selective reporting and others. Risks in each domain were assessed in the following three categories: high risk, low risk and unclear. All disagreements were resolved as the consensus decision of all four researchers.

\section{Statistical analysis}

The results were analyzed using Review Manager (RevMan) version 5.3 software (The Cochrane Collaboration, Oxford, UK). For dichotomous outcomes, results are expressed as risk ratio (RR) with 95\% confidence interval (CI). Changes in eGFR are expressed as the mean difference (MD). Values of eGFR and the standard deviation were calculated according to the Cochrane Handbook for Systemic Review and the method described by Follmann et al. [10], if those data were not provided in the publication. Heterogeneity across included studies was assessed statistically by calculating the overall $I^{2}$ values, with $I^{2}$ values of $<25 \%, 25 \%$ to $<75 \%$ and $\geq 75 \%$ corresponding to low, moderate and high levels of heterogeneity, respectively [11]. We performed subgroup analyses according 


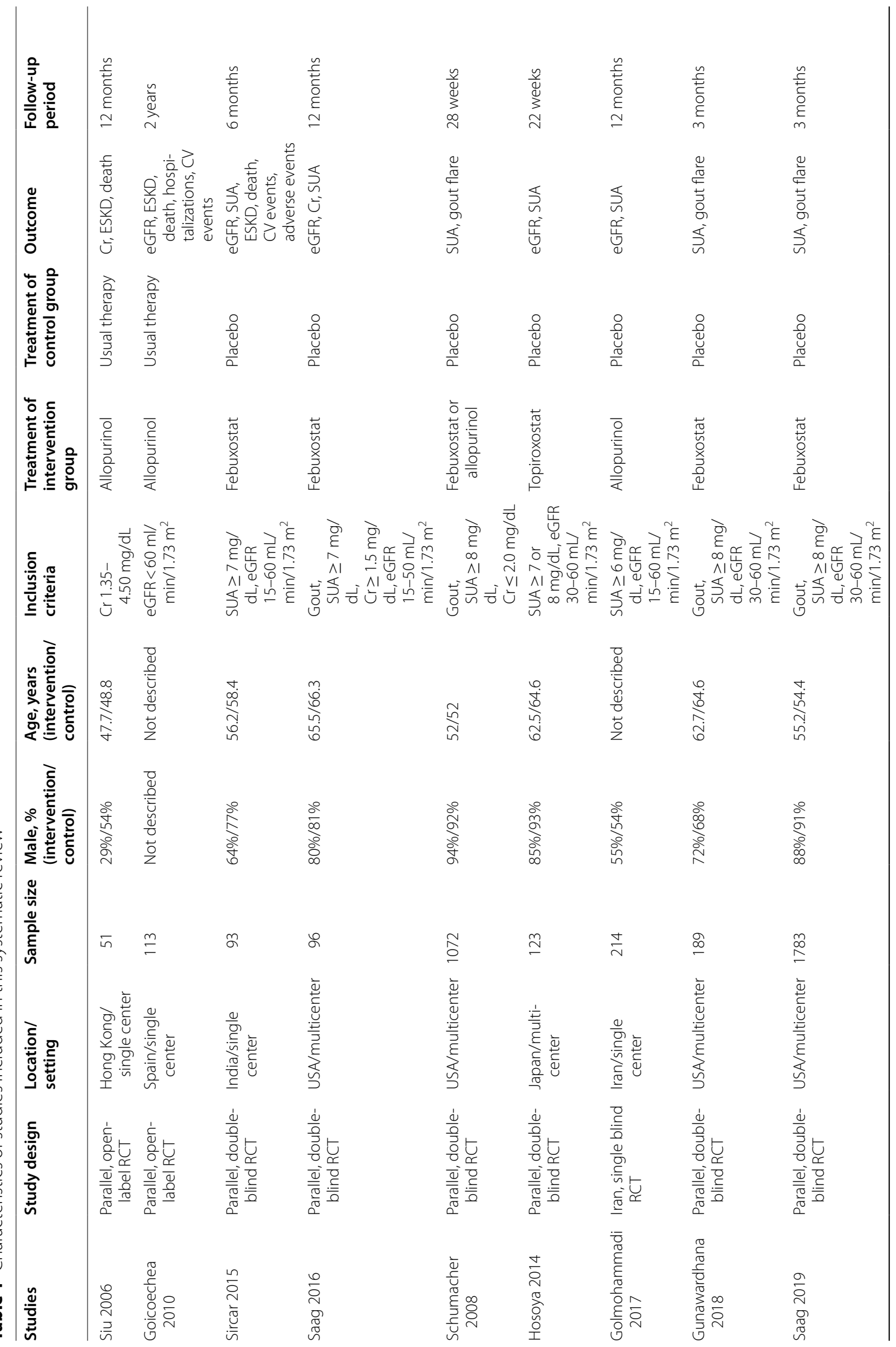




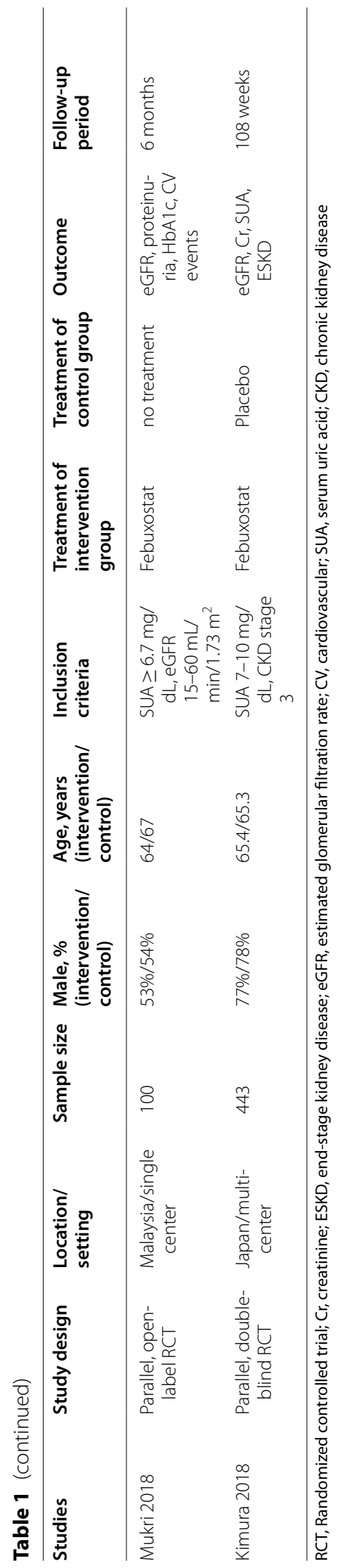


Table 2 Risk of bias summary

\begin{tabular}{|c|c|c|c|c|c|c|c|}
\hline Study & $\begin{array}{l}\text { Random } \\
\text { sequence } \\
\text { generation }\end{array}$ & $\begin{array}{l}\text { Allocation } \\
\text { concealment }\end{array}$ & $\begin{array}{l}\text { Blinding of } \\
\text { participants and } \\
\text { personnel }\end{array}$ & $\begin{array}{l}\text { Blinding of } \\
\text { outcome } \\
\text { assessment }\end{array}$ & $\begin{array}{l}\text { Incomplete } \\
\text { outcome } \\
\text { data }\end{array}$ & Selective reporting & Other bias \\
\hline Siu 2006 & Low & Unclear & High & Unclear & Low & Unclear & Low \\
\hline Goicoechea 2010 & Low & Unclear & High & Low & Low & Unclear & Low \\
\hline Sircar 2015 & Low & Low & Low & Unclear & High & Low & Low \\
\hline Saag 2016 & Unclear & Unclear & Low & Unclear & High & Unclear & Low \\
\hline Schumacher 2008 & Unclear & Unclear & Unclear & Unclear & High & Unclear & Low \\
\hline Hosoya 2014 & Low & Unclear & Unclear & Unclear & Low & Low & Low \\
\hline Golmohammadi 2017 & Unclear & Unclear & Unclear & Unclear & Unclear & Unclear & Low \\
\hline Gunawardhana 2018 & Low & Unclear & Low & Unclear & High & Unclear & Low \\
\hline Saag 2019 & Low & Unclear & Low & Unclear & High & Low & Low \\
\hline Mukri 2018 & Low & Unclear & High & Unclear & Low & Unclear & Low \\
\hline Kimura 2018 & Low & Unclear & Unclear & Unclear & Low & Low & Low \\
\hline
\end{tabular}

to the possible sources of heterogeneity including type of ULT, follow-up period and baseline eGFR. A funnel plot was used to assess potential publication bias. Data were pooled using random-effects modeling.

\section{Results}

\section{Study selection}

Figure 1 summarizes the search strategy used. The initial search yielded 167 articles, of which 137 articles were excluded by reviewing the title and abstract alone. A total of 30 articles underwent full-length review, of which 11 studies were included in the qualitative analysis [12-22].

\section{Study characteristics}

Details of the 11 studies, including a total of 4,277 CKD patients, are summarized in Table 1 [12-22]. Drugs used in the intervention groups of all studies were XOR inhibitors (allopurinol, febuxostat or topiroxostat). Six studies were from Asia [12, 14, 17, 18, 21, 22], 4 from North America [15, 16, 19, 20] and 1 from Europe [13]. Intervention groups received febuxostat in 7 studies [14-16, 19-22], allopurinol in 3 studies [12, 13, 18] and topiroxostat in 1 study [17]. Control groups received placebo in 8 studies [14-20,22], usual therapy in 2 studies [12, 13] and no treatment in 1 study [21]. Follow-up periods were $<1$ year in 6 studies $[14,16,17$,
19-21] and $\geq 1$ year in 5 studies $[12,13,15,18,22]$. Percentage of male participants, mean age and percentage of diabetic patients varied from $24 \%$ [12] to $100 \%$ [21], from 48 [12] to 67 years [15, 21] and from $24 \%$ [12] to $100 \%$ [21], respectively. In addition, serum UA at baseline varied from 7.3 [13] to $10.8 \mathrm{mg} / \mathrm{dL}$ [15]. The quality of studies is shown in Table 2. Several studies showed a high risk of bias due to inadequate blinding of participants and personnel and incomplete outcome data. Other components of risk of bias were not severe.

\section{Changes in eGFR}

Results from the five studies were in favor of ULT, but no significant differences were identified (MD, 2.52; $95 \%$ CI, -0.15 to 5.18 ; Fig. 2a) $[13,14,17,21,22]$. In this comparison, a high level of heterogeneity was detected $\left(\tau^{2}=8.04, \chi^{2}=83.71, I^{2}=95 \%\right)$. We performed subgroup analyses according to the possible sources of heterogeneity. One study used allopurinol as treatment for the intervention group and the result was in favor of ULT treatment (MD, 4.60; 95\%CI, 4.11-5.09; Fig. 2b). Four studies used non-purine structure agents, including febuxostat and topiroxostat, as treatment for the intervention group and showed no significant difference in eGFR changes (MD, 1.46 ; $95 \% \mathrm{CI},-0.40$ to

\footnotetext{
(See figure on next page.)

Fig. 2 Forest plot comparing changes in estimated glomerular filtration rate (eGFR) between uric acid-lowering therapy (ULT) users and nonusers: a all studies; and subgroup analysis of b study used allopurinol; c studies used non-purine structure agents, including febuxostat and topiroxostat; d studies with follow-up period of longer than or equal to 1 year; e studies with follow-up period of less than 1 year; $\mathbf{f}$ studies including patients with eGFR of greater than or equal to $40 \mathrm{~mL} / \mathrm{min} / 1.73 \mathrm{~m}^{2}$; and $\mathbf{g}$ studies including patients with eGFR of less than $40 \mathrm{~mL} / \mathrm{min} / 1.73 \mathrm{~m}$; $\mathbf{h}$ forest plot comparing incidence of end-stage kidney disease (ESKD) between ULT users and nonusers; and $\mathbf{i}$ forest plot comparing incidence of treatment-emergent adverse events (TEAEs) leading to study drug discontinuation between ULT users and nonusers. Abbreviations: ULT, uric acid-lowering therapy
} 


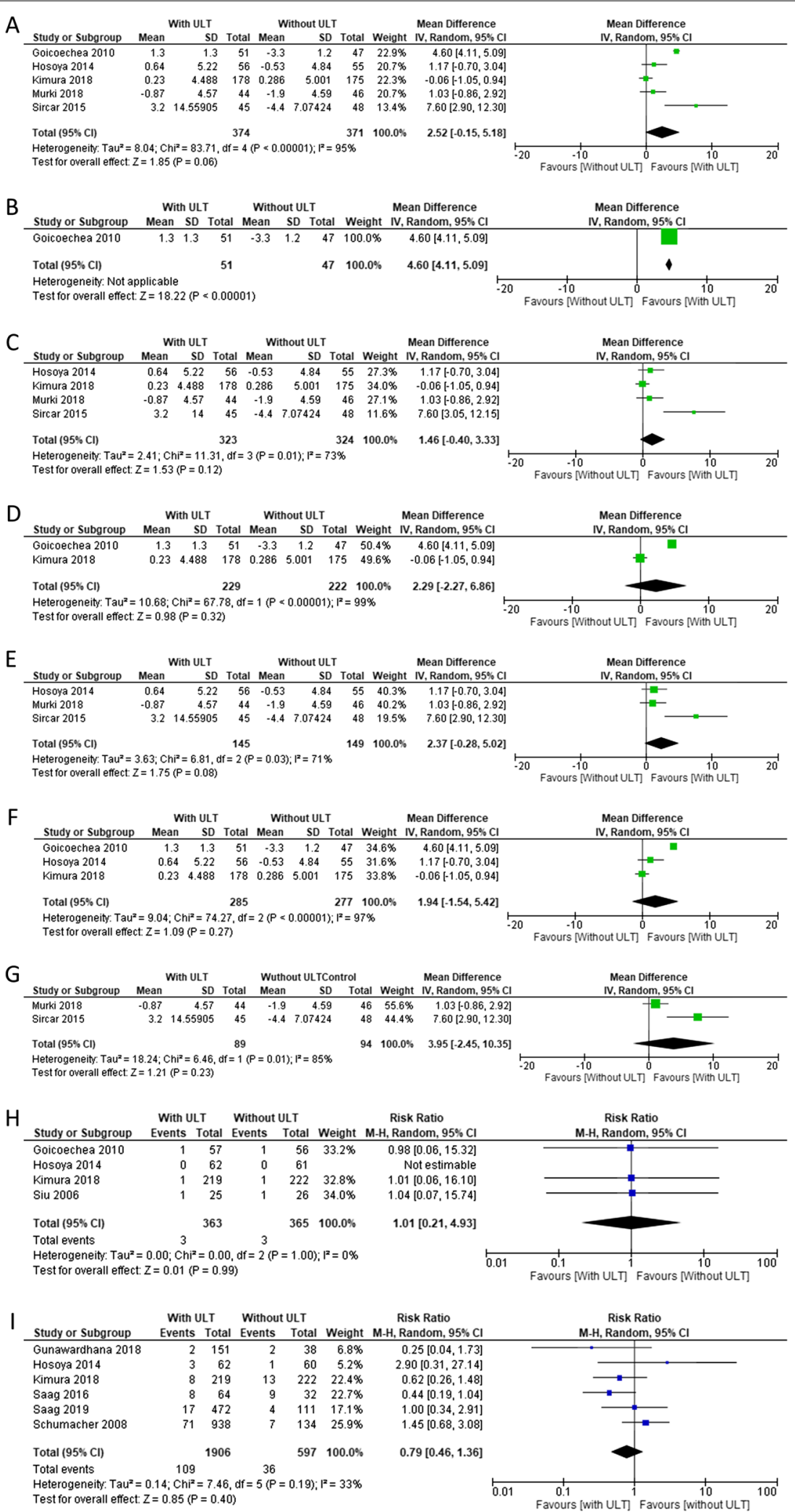

Fig. 2 (See legend on previous page.) 


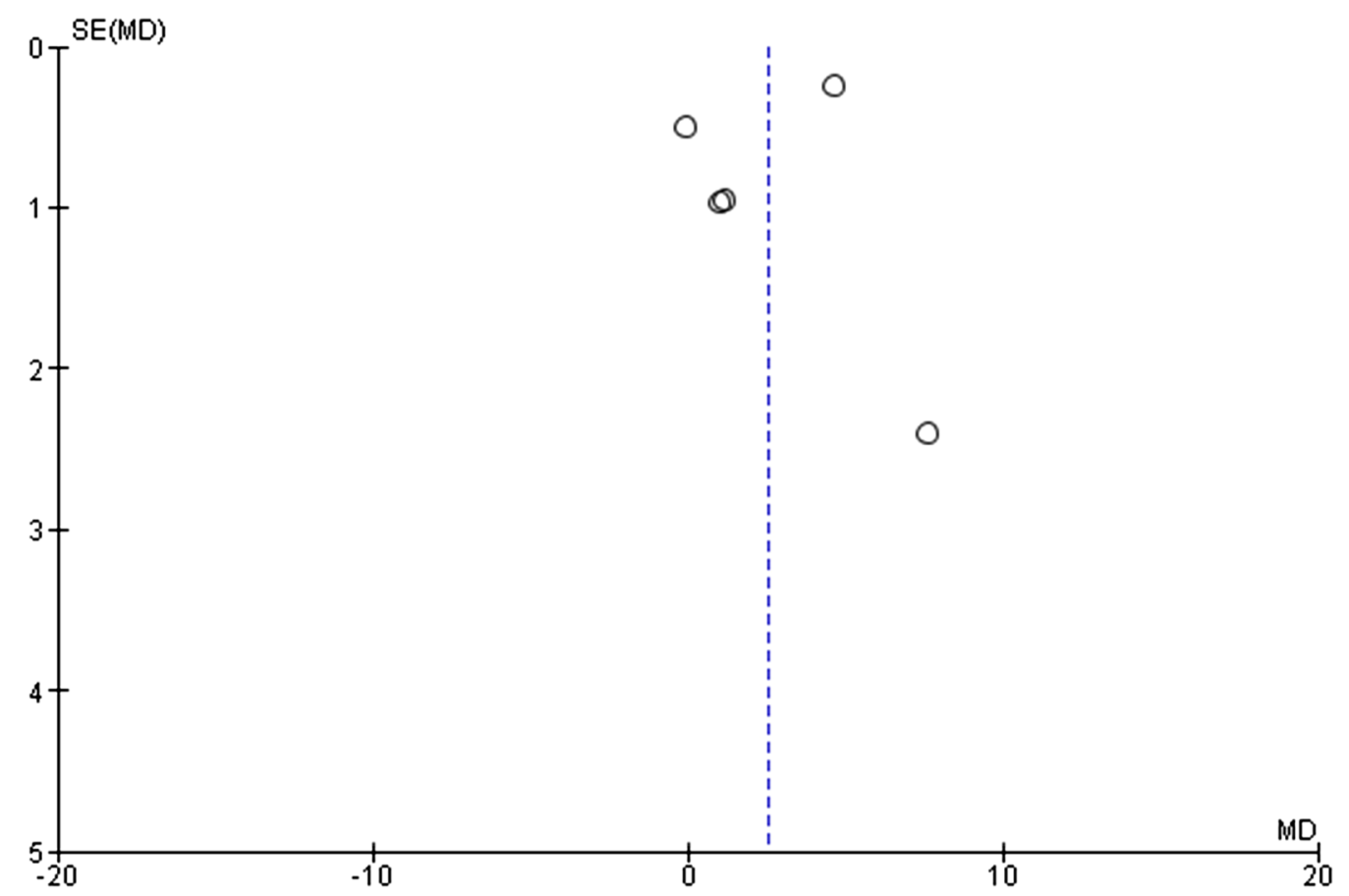

Fig. 3 Funnel plot of studies evaluating potential publication bias

3.33; Fig. 2c). The effect of ULT on changes in eGFR was similar between two groups according to follow-up period (MD 2.29; 95\%CI, -2.27 to 6.86 and MD 2.37; $95 \% \mathrm{CI},-0.28$ to 5.02 for studies with follow-up period of longer than or equal to 1 year and less than 1 year; Fig. 2d and e) and baseline eGFR (MD 1.94; 95\%CI, -1.54 to 0.94 and MD 3.95; $95 \% \mathrm{CI},-2.45$ to 10.35 for studies including patients with eGFR of greater than or equal to $40 \mathrm{~mL} / \mathrm{min} / 1.73 \mathrm{~m}^{2}$ and less than $40 \mathrm{~mL} /$ $\mathrm{min} / 1.73 \mathrm{~m}^{2}$; Fig. $2 \mathrm{f}$ and g). A funnel plot for eGFR showed no publication bias (Fig. 3).

\section{Incidence of ESKD}

The incidence of ESKD, assessed in four studies, was extremely low and similar between ULT users and nonusers (RR, 1.01; 95\%CI, 0.21-4.93; Fig. 2h). Heterogeneity was not detected in this comparison $\left(\tau^{2}=0.00, \chi^{2}=0.00\right.$, $I^{2}=0 \%$.

\section{Adverse events}

No significant difference in the incidence of TEAEs leading to study drug discontinuation was apparent between ULT users and nonusers (RR, 0.79; 95\%CI, 0.46-1.36; Fig. 2i). A moderate level of heterogeneity was detected in this comparison $\left(\tau^{2}=0.14, \chi^{2}=7.46, I^{2}=33 \%\right)$.

\section{Discussion}

This SR and meta-analysis of 11 studies with 4,277 CKD patients was conducted to clarify the effectiveness of ULT [12-22]. Although patients with ULT tended to show superior preservation of eGFR as compared to those without ULT, no significant difference was identified. As expected, no significant differences in the incidences of ESKD or TEAEs leading to drug discontinuation were noted between ULT users and nonusers, probably due to the extremely lower incidences of these outcomes. These data support the findings of previous SRs $[4,5]$. Although there have been several studies including CKD patients, its definition varied according to the studies, and the presence of diabetes, proteinuria and serum creatinine in addition to eGFR were taken into account for diagnosis. In caring for CKD patients with hyperuricemia, eGFR is the most important factor because it directly affects drug administration and dose in a clinical setting. Additionally, eGFR is independent determinant of risk of death and the development of CVD in CKD patients. To the best of our knowledge, this was the first SR only among CKD patients whose eGFR was less than $60 \mathrm{~mL} / \mathrm{min} / 1.73 \mathrm{~m}^{2}$.

Whether lowering UA concentrations or inhibiting XOR offers the best improvements in patient outcomes (including mortality rate, incidence of CVD and preservation of renal function) remains contentious. UA is well known to 
induce endothelial dysfunction, mediated by local activation of oxidative stress and the renin-angiotensin system $[1,23]$. In addition, hyperuricemia provokes arteriolopathy of the glomerular vessels, impairing the autoregulatory response of afferent arterioles and resulting in glomerular hypertension [24]. ULT would thus be predicted to prevent the incidence of CVD through the suppression of arteriosclerosis. On the other hand, XOR activity was increased in patients with coronary disease and could enhance the atherosclerotic process through promoting endothelial dysfunction [25]. A randomized, placebocontrolled trial by George et al. [26] found that allopurinol significantly increased endothelial function as assessed by forearm blood flow response to acetylcholine, whereas the uricosuric agent probenecid had no effect, despite similar UA-lowering effects. To date, inhibition of XOR has been assumed to reduce oxidative stress through reductions in the production of reactive oxygen species [27, 28]. Kato et al. [29] recently reported that ATP enhancement through enhanced purine salvage is the more likely mechanism of the beneficial effects of XOR inhibitors. In addition, these mechanisms are thought to be associated with renoprotective effect of XOR inhibitors.

We found only one study comparing changes in eGFR between patients with and without allopurinol [13]. Goicoechea et al. [13] conducted a prospective, randomized trial of 113 CKD patients, and found that eGFR was increased in the allopurinol group, but decreased in the control group after 24 months. However, some baseline characteristics differed between groups in that study. In particular, baseline UA levels were higher in the intervention group than in the control group. Since treatment with allopurinol is associated with a risk of hypersensitivity syndrome [30] and Stevens-Johnson syndrome [31], particularly among patients with renal impairment, clinicians often hesitate to use this agent for CKD patients. Two recent, large-scale, double-blinded, multicenter, randomized, placebo-controlled clinical trials have tested the renoprotective effects of allopurinol among renal patients [32, 33]. Doria et al. [32] conducted the Preventing Early Renal Loss in Diabetes (PERL) trial of 530 patients with type 1 diabetes who had stage 1-3 CKD. Badve et al. [33] conducted the Controlled Trial of Slowing of Kidney Disease Progression from the Inhibition of Xanthine Oxidase (CKD-FIX), including 369 patients with stage 3 or 4 CKD showing rapid declines in eGFR or clinically significant proteinuria. Both RCTs failed to show that treatment with allopurinol resulted in slower declines in eGFR compared to placebo. Recent SR also demonstrated that ULT did not produce benefits on the clinical outcomes, including major adverse cardiovascular events, all-cause mortality and kidney failure. Nevertheless, ULT attenuated the decline in the slope of eGFR. However, this study included not only CKD but also non-CKD patients. In contrast, we included only CKD patients defined as eGFR $<60 \mathrm{~mL} / \mathrm{min} / 1.73 \mathrm{~m}^{2}$ [34].

We could not confirm any eGFR-preserving effects of treatment with the newer XOR inhibitors, febuxostat and topiroxostat. Kimura et al. [22] reported that the slope of eGFR did not differ significantly between CKD patients with and without febuxostat in a multicenter, randomized, double-blinded, placebo-controlled trial of 467 patients with stage 3 CKD and asymptomatic hyperuricemia. In addition, Hosoya et al. [17] reported that changes in eGFR did not differ significantly between CKD patients with and without topiroxostat in a 22-week randomized, multicenter, double-blinded study. However, the change in urinary albumin-to-creatinine ratio was decreased in the topiroxostat group as compared to the placebo group. Several reports have indicated that XOR inhibitors decrease the exacerbation of albuminuria or proteinuria, which would risk progression of renal disease, among patients with normal renal function [35, 36] and CKD patients [17, 37-39].

The present study had several limitations. There was marked heterogeneity in this meta-analysis because of several differences in background of each study. Especially, follow-up periods varied widely from 3 months to 2 years and the changes in renal function were thus difficult to interpret. Additionally, studies with a longer observational period are needed to clarify the effect of ULT on the changes in eGFR. Several retrospective cohort studies assessed long-term changes in eGFR among CKD patients with ULT, and SR including these observational studies revealed that use of febuxostat was associated with higher eGFR and reduced risk for renal disease progression [40]. Unfortunately, the possible causes of heterogeneity were not clarified despite several subgroup analyses. On the other hand, the strength of the present study was the first SR including only patients with eGFR, most important factor in the CKD management, lower than $60 \mathrm{~mL} / \mathrm{min} / 1.73 \mathrm{~m}^{2}$.

\section{Conclusions}

In the present SR of 11 RCTs with 4277 CKD patients, patients with ULT tended to show superior effects in preservation of eGFR as compared to those without ULT, but no significant differences were evident. No significant differences in the incidence of ESKD or TEAEs leading to study drug discontinuation were found between ULT users and nonusers. XOR inhibitors could be effective for improving outcomes in CKD patients. Further studies in hyperuricemic CKD patients should be undertaken to verify the renoprotective effects of ULT, especially newer XOR inhibitors such as febuxostat and topiroxostat. 


\begin{abstract}
Abbreviations
CVD: Cardiovascular disease; UA: Uric acid; CKD: Chronic kidney disease; ESKD: End-stage kidney disease; RCT: Randomized controlled trial; SR: Systematic review; eGFR: Estimated glomerular filtration rate; ULT: Uric acid-lowering therapy; XOR: Xanthine oxidoreductase; PRISMA: The preferred reporting items for systematic reviews and meta-analyses; TEAE: Treatment-emergent adverse events; RR: Risk ratio; Cl: Confidence interval; MD: Mean difference; PERL: The Preventing Early Renal Loss in Diabetes; CKD-FIX: The controlled trial of slowing of kidney disease progression from the inhibition of xanthine oxidase.
\end{abstract}

\section{Supplementary Information}

The online version contains supplementary material available at https://doi. org/10.1186/s41100-021-00363-7.

\section{Additional file 1. PRISMA 2009 checklist \\ Additional file 2 . Search strategy}

\section{Acknowledgements}

We wish to thank Dr. Yasuto Sato from the Department of Public Health, Tokyo Women's Medical University, Tokyo, Japan and Dr. Toshio Morizane from The Japan Council for Quality Health Care for their technical support regarding the management of the systematic review.

\section{Authors' contributions}

$\mathrm{YM}, \mathrm{KI}$ and $\mathrm{SU}$ were involved in concept and design of the study. YM, TK, NS, SY, KI and SU helped in analysis and interpretation of data. YM, TK, NS and SY was involved in screening and selection of relevant studies, quality assessment. YM and SU contributed to writing of original draft. YM, TK, NS, SY, KI and SU helped in reviewing and editing of manuscript. All authors read and approved the final manuscript.

\section{Funding}

This research received no specific grant from any funding agency in the public, commercial or not-for-profit sectors.

\section{Availability of data and materials}

The datasets used and/or analyzed during the current study are available from the corresponding author on reasonable request.

\section{Declarations}

\section{Ethics approval and consent to participate}

Not applicable.

\section{Consent for publication}

Not applicable.

\section{Competing interests}

Y.M. received honoraria from Sanwa Kagaku Kenkyusho Co., Ltd. K.I. received honoraria from Fuji Yakuhin Co., Ltd. and Teijin Pharma, Ltd. S.U. received honoraria from Sanwa Kagaku Kenkyusho Co., Ltd., Fuji Yakuhin Co., Ltd. and Teijin Pharma, Ltd. No other authors have any conflicts of interest to declare.

\section{Author details}

'Division of Nephrology and Hypertension, Department of Internal Medicine, The Jikei University School of Medicine, 3-19-18 Nishi-shinbashi, Minato-ku, Tokyo 105-8471, Japan. ${ }^{2}$ Division of Nephrology, Department of Internal Medicine, Teikyo University School of Medicine, Tokyo, Japan. ${ }^{3}$ Dialysis Center, Atami Oceanview Hospital, Shizuoka, Japan. ${ }^{4}$ Department of Pathophysiology, Tokyo University of Pharmacy and Life Sciences, Tokyo, Japan. ${ }^{5}$ International Exchange Center, Teikyo Heisei University, Tokyo, Japan.

Received: 7 April 2021 Accepted: 10 August 2021

Published online: 26 August 2021
References

1. Feig DI, Kang DH, Johnson RJ. Uric acid and cardiovascular risk. N Engl J Med. 2008;359(17):1811-21.

2. Feig DI. Uric acid: a novel mediator and marker of risk in chronic kidney disease? Curr Opin Nephrol Hypertens. 2009;18(6):526-30.

3. Hsu CY, Iribarren C, McCulloch CE, Darbinian J, Go AS. Risk factors for end-stage renal disease: 25-year follow-up. Arch Intern Med. 2009;169(4):342-50.

4. Sampson AL, Singer RF, Walters GD. Uric acid lowering therapies for preventing or delaying the progression of chronic kidney disease. Cochrane Database Syst Rev. 2017;10:CD009460.

5. Bose B, Badve SV, Hiremath SS, Boudville N, Brown FG, Cass A, et al. Effects of uric acid-lowering therapy on renal outcomes: a systematic review and meta-analysis. Nephrol Dial Transpl. 2014;29(2):406-13.

6. Kanji T, Gandhi M, Clase CM, Yang R. Urate lowering therapy to improve renal outcomes in patients with chronic kidney disease: systematic review and meta-analysis. BMC Nephrol. 2015;16:58.

7. Liu X, Zhai T, Ma R, Luo C, Wang H, Liu L. Effects of uric acid-lowering therapy on the progression of chronic kidney disease: a systematic review and meta-analysis. Ren Fail. 2018;40(1):289-97.

8. Lin TC, Hung LY, Chen YC, Lo WC, Lin CH, Tam KW, et al. Effects of febuxostat on renal function in patients with chronic kidney disease: A systematic review and meta-analysis. Medicine. 2019;98(29):e16311.

9. Moher D, Liberati A, Tetzlaff J, Altman DG, Group P. Preferred reporting items for systematic reviews and meta-analyses: the PRISMA statement. PLOS Med. 2009;6(7):e1000097.

10. Follmann D, Elliott P, Suh I, Cutler J. Variance imputation for overviews of clinical trials with continuous response. J Clin Epidemiol. 1992;45(7):769-73.

11. Higgins JP, Thompson SG, Deeks JJ, Altman DG. Measuring inconsistency in meta-analyses. BMJ. 2003;327(7414):557-60.

12. Siu YP, Leung KT, Tong MK, Kwan TH. Use of allopurinol in slowing the progression of renal disease through its ability to lower serum uric acid level. Am J Kidney Dis. 2006;47(1):51-9.

13. Goicoechea M, de Vinuesa SG, Verdalles U, Ruiz-Caro C, Ampuero J, Rincon A, et al. Effect of allopurinol in chronic kidney disease progression and cardiovascular risk. Clin J Am Soc Nephrol. 2010;5(8):1388-93.

14. Sircar D, Chatterjee S, Waikhom R, Golay V, Raychaudhury A, Chatterjee S, et al. Efficacy of febuxostat for slowing the GFR decline in patients with CKD and asymptomatic hyperuricemia: a 6-month, double-blind, randomized. Placebo-Controlled Trial Am J Kidney Dis. 2015;66(6):945-50.

15. Saag KG, Whelton A, Becker MA, MacDonald P, Hunt B, Gunawardhana L. Impact of febuxostat on renal function in gout patients with moderateto-severe renal impairment. Arthritis Rheumatol. 2016;68(8):2035-43.

16. Schumacher HR Jr, Becker MA, Wortmann RL, Macdonald PA, Hunt $B$, Streit J, et al. Effects of febuxostat versus allopurinol and placebo in reducing serum urate in subjects with hyperuricemia and gout: a 28-week, phase III, randomized, double-blind, parallel-group trial. Arthritis Rheumatol. 2008;59(11):1540-8.

17. Hosoya T, Ohno I, Nomura S, Hisatome I, Uchida S, Fujimori S, et al. Effects of topiroxostat on the serum urate levels and urinary albumin excretion in hyperuricemic stage 3 chronic kidney disease patients with or without gout. Clin Exp Nephrol. 2014;18(6):876-84.

18. Golmohammadi S, Almasi A, Manouchehri M, Omrani HR, Zandkarimi MR. Allopurinol against progression of chronic kidney disease. Iran J Kidney Dis. 2017;11(4):286-93.

19. Gunawardhana L, Becker MA, Whelton A, Hunt B, Castillo M, Saag K. Efficacy and safety of febuxostat extended release and immediate release in patients with gout and moderate renal impairment: phase II placebocontrolled study. Arthritis Res Ther. 2018;20(1):99.

20. Saag KG, Becker MA, Whelton A, Hunt B, Castillo M, Kisfalvi K, et al. Efficacy and safety of febuxostat extended and immediate release in patients with gout and renal impairment: a phase III Placebo-Controlled Study. Arthritis Rheumatol. 2019;71(1):143-53.

21. Mukri MNA, Kong WY, Mustafar R, Shaharir SS, Shah SA, Abdul Gafor $\mathrm{AH}$, et al. Role of febuxostat in retarding progression of diabetic kidney disease with asymptomatic hyperuricemia: a 6-months open-label, randomized controlled trial. EXCLI J. 2018;17:563-75.

22. Kimura K, Hosoya T, Uchida S, Inaba M, Makino H, Maruyama S, et al. Febuxostat therapy for patients with stage 3 CKD and asymptomatic hyperuricemia: a randomized trial. Am J Kidney Dis. 2018;72(6):798-810. 
23. Yu MA, Sanchez-Lozada LG, Johnson RJ, Kang DH. Oxidative stress with an activation of the renin-angiotensin system in human vascular endothelial cells as a novel mechanism of uric acid-induced endothelial dysfunction. J Hypertens. 2010;28(6):1234-42.

24. Sanchez-Lozada LG, Tapia E, Santamaria J, Avila-Casado C, Soto V, Nepomuceno T, et al. Mild hyperuricemia induces vasoconstriction and maintains glomerular hypertension in normal and remnant kidney rats. Kidney Int. 2005;67(1):237-47.

25. Spiekermann S, Landmesser U, Dikalov S, Bredt M, Gamez G, Tatge H, et al. Electron spin resonance characterization of vascular xanthine and NAD(P) $\mathrm{H}$ oxidase activity in patients with coronary artery disease: relation to endothelium-dependent vasodilation. Circulation. 2003;107(10):1383-9.

26. George J, Carr E, Davies J, Belch JJ, Struthers A. High-dose allopurinol improves endothelial function by profoundly reducing vascular oxidative stress and not by lowering uric acid. Circulation. 2006;114(23):2508-16.

27. Ryan MJ, Jackson JR, Hao Y, Leonard SS, Alway SE. Inhibition of xanthine oxidase reduces oxidative stress and improves skeletal muscle function in response to electrically stimulated isometric contractions in aged mice. Free Radic Biol Med. 2011;51(1):38-52.

28. Cantu-Medellin N, Kelley EE. Xanthine oxidoreductase-catalyzed reactive species generation: a process in critical need of reevaluation. Redox Biol. 2013;1:353-8.

29. Kato S, Kato M, Kusano T, Nishino T. New strategy that delays progression of amyotrophic lateral sclerosis in G1H-G93A transgenic mice: oral administration of xanthine oxidoreductase inhibitors that are not substrates for the purine salvage pathway. J Neuropathol Exp Neurol. 2016:75(12):1124-44.

30. Ramasamy SN, Korb-Wells CS, Kannangara DR, Smith MW, Wang N, Roberts DM, et al. Allopurinol hypersensitivity: a systematic review of all published cases, 1950-2012. Drug Saf. 2013;36(10):953-80.

31. Halevy S, Ghislain PD, Mockenhaupt M, Fagot JP, Bouwes Bavinck JN Sidoroff A, et al. Allopurinol is the most common cause of Stevens-Johnson syndrome and toxic epidermal necrolysis in Europe and Israel. J Am Acad Dermatol. 2008;58(1):25-32.

32. Doria A, Galecki AT, Spino C, Pop-Busui R, Cherney DZ, Lingvay I, et al. Serum urate lowering with allopurinol and kidney function in type 1 diabetes. N Engl J Med. 2020;382(26):2493-503.
33. Badve SV, Pascoe EM, Tiku A, Boudville N, Brown FG, Cass A, et al. Effects of allopurinol on the progression of chronic kidney disease. N Engl J Med. 2020;382(26):2504-13.

34. Chen Q, Wang Z, Zhou J, Chen Z, Li Y, Li S, et al. Effect of urate-lowering therapy on cardiovascular and kidney outcomes: a systematic review and meta-analysis. Clin J Am Soc Nephrol. 2020;15(11):1576-86.

35. Kojima S, Matsui K, Hiramitsu S, Hisatome I, Waki M, Uchiyama K, et al. Febuxostat for cerebral and cardiorenovascular events prevention study. Eur Heart J. 2019;40(22):1778-86.

36. Liu P, Chen Y, Wang B, Zhang F, Wang D, Wang Y. Allopurinol treatment improves renal function in patients with type 2 diabetes and asymptomatic hyperuricemia: 3-year randomized parallel-controlled study. Clin Endocrinol. 2015;83(4):475-82.

37. Sezai A, Soma M, Nakata K, Osaka S, Ishii Y, Yaoita H, et al. Comparison of febuxostat and allopurinol for hyperuricemia in cardiac surgery patients with chronic kidney disease (NU-FLASH trial for CKD). J Cardiol. 2015;66(4):298-303.

38. Mizukoshi T, Kato S, Ando M, Sobajima H, Ohashi N, Naruse T, et al. Renoprotective effects of topiroxostat for Hyperuricaemic patients with overt diabetic nephropathy study (ETUDE study): a prospective, randomized, multicentre clinical trial. Nephrology (Carlton). 2018;23(11):1023-30.

39. Tanaka K, Nakayama M, Kanno M, Kimura H, Watanabe K, Tani Y, et al. Renoprotective effects of febuxostat in hyperuricemic patients with chronic kidney disease: a parallel-group, randomized, controlled trial. Clin Exp Nephrol. 2015;19(6):1044-53.

40. Hu AM, Brown JN. Comparative effect of allopurinol and febuxostat on long-term renal outcomes in patients with hyperuricemia and chronic kidney disease: a systematic review. Clin Rheumatol. 2020;39(11):3287-94.

\section{Publisher's Note}

Springer Nature remains neutral with regard to jurisdictional claims in published maps and institutional affiliations.
Ready to submit your research? Choose BMC and benefit from:

- fast, convenient online submission

- thorough peer review by experienced researchers in your field

- rapid publication on acceptance

- support for research data, including large and complex data types

- gold Open Access which fosters wider collaboration and increased citations

- maximum visibility for your research: over $100 \mathrm{M}$ website views per year

At BMC, research is always in progress.

Learn more biomedcentral.com/submissions 